\title{
Revista Colombiana de

\section{Patrones de prescripción de los nuevos anticoagulantes orales y sus costos económicos en Colombia}

\author{
Jorge E. Machado-Alba ${ }^{a, *}$, Santiago García-Betancur ${ }^{a}$, Federico Villegas-Cardona ${ }^{b}$ \\ y Diego Alejandro Medina-Morales ${ }^{a}$
}

\author{
a Grupo de Investigación en Farmacoepidemiología y Farmacovigilancia, Universidad Tecnológica de Pereira-Audifarma, S.A., \\ Pereira, Colombia \\ ${ }^{\mathrm{b}}$ Asociación Colombiana Médica Estudiantil (ACOME)
}

Recibido el 11 de mayo de 2015; aceptado el 23 de agosto de 2015

Disponible en Internet el 9 de octubre de 2015

\author{
PALABRAS CLAVE \\ Anticoagulantes; \\ Dabigatrán; \\ Rivaroxabán; \\ Apixabán; \\ Farmacoepidemiología; \\ Atención primaria; \\ Colombia
}

\begin{abstract}
Resumen
Introducción: Durante décadas los antagonistas de la vitamina $\mathrm{K}$ fueron los medicamentos disponibles para el manejo del paciente que requería anticoagulación. Los llamados nuevos anticoagulantes orales, con aparente mejor perfil de seguridad han sido recientemente aprobados para algunas indicaciones, sin embargo, en Colombia es escasa la información acerca del patrón de uso de estos medicamentos.

Objetivo: Determinar los patrones de prescripción de los nuevos anticoagulantes orales y variables asociadas a su uso en una población de pacientes afiliados al Sistema General de Seguridad Social en Salud (SGSSS) de Colombia durante el año 2014.

Métodos: Un estudio observacional de corte transversal. Se seleccionaron pacientes con dispensación del dabigatran, del rivaroxaban y del apixaban durante el trimestre febrero-abril de 2014, de una base de datos de 6,5 millones de afiliados al SGSSS. Se midieron variables sociodemográficas, farmacológicas, de comedicación (comorbilidades) y económicas. Se utilizaron pruebas t de Student, Chi cuadrado y modelos de regresión logística.

Resultados: Un total de 1.310 pacientes consumieron nuevos anticoagulantes orales, con predominio masculino $(57,2 \%)$, en monoterapia $(88,0 \%$ y la asociación con antiagregantes se presentó en el $10,5 \%$. El agente más prescrito fue el rivaroxaban (52,9\%). El costo por 1.000 habitantes/día del dabigatran COP $\$ 334,6$, del rivaroxaban COP $\$ 930,7$ y del apixaban COP $\$ 32,6$. El 49,1\% de pacientes tenía diagnóstico de fibrilación auricular.

Conclusiones: El rivaroxaban fue el medicamento con mayor costo. A partir de estos patrones se puede determinar que los nuevos anticoagulantes orales se están empleando principalmente
\end{abstract}

\footnotetext{
* Autor para correspondencia.

Correo electrónico: machado@utp.edu.co (J.E. Machado-Alba).
} 


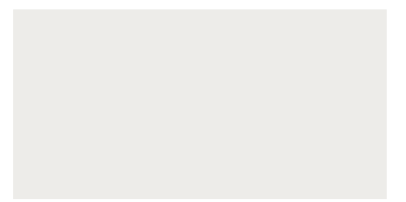

\section{KEYWORDS}

Blood-thinning drugs;

Dabigatran;

Rivaroxaban;

Aprixaban;

Pharmacoepidemiology;

Primary care;

Colombia en monoterapia. Se requieren nuevos estudios que evalúen la efectividad y la seguridad en la población colombiana.

(c) 2015 Sociedad Colombiana de Cardiología y Cirugía Cardiovascular. Publicado por Elsevier España, S.L.U. Este es un artículo Open Access bajo la licencia CC BY-NC-ND (http:// creativecommons.org/s/by-nc-nd/4.0/).

\section{Prescription patterns of new oral blood thinning drugs and their economic impact in Colombia}

\begin{abstract}
Introduction: For decades, vitamin $\mathrm{K}$ antagonists were the drugs available for management of patients requiring anticoagulation therapy. The so-called new oral blood thinners, with an apparently better safety profile, have been approved for some cases; however, Colombia still lacks the information on prescription patterns for these drugs.

Objetive: To determine prescription patterns of new oral blood-thinning drugs and the variables associated to their intake in a group of patients affiliated to the Colombian General Health and Social Security System (SGSSS) during 2014.

Methods: Cross-sectional observational study. Patients taking dabigatran, rivaroxaban and apixaban during the February-April 2014 quarter were selected from the database of 6.5 SGSSS affiliated members. Sociodemographic, pharmacological, co-medication (comorbidities) and economic variables were assessed. Student's t test, chi-square test and logistic regression models were used.

Results: A total of 1,310 patients were administered new oral blood thinners, with a male predominance $(57.2 \%)$, in single-drug therapy (88\%), and an association with antiplatelet drugs was present in $10.5 \%$ of the cases. The most commonly prescribed medication was rivaroxaban (52.9\%). The cost per 1,000 people/day of dabigatran COL $\$ 334.6$, of rivaroxaban COL $\$ 930.7$ and apixaban COL $\$ 32.6 .49 .1 \%$ of the patients had a diagnosis of atrial fibrillation.

Conclusions: Rivaroxaban was the medication with the greater cost. Based on these patterns it can be determines that the new oral blood-thinning drugs are being used mainly as a single-drug therapy. More studies assessing the efficacy and safety profile in Colombian population need to be conducted.

(c) 2015 Sociedad Colombiana de Cardiología y Cirugía Cardiovascular. Published by Elsevier España, S.L.U. This is an open access article under the CC BY-NC-ND license (http:// creativecommons.org/licenses/by-nc-nd/4.0/).
\end{abstract}

\section{Introducción}

Durante muchas décadas, los antagonistas de la vitamina $\mathrm{K}$ fueron los únicos medicamentos disponibles para el uso en prevención primaria y secundaria de eventos tromboembólicos venosos y arteriales, mostrando ser altamente efectivos en muchos escenarios clínicos que requieren anticoagulación por 3 o más meses, como la fibrilación auricular (FA), la tromboembolia venosa (TEV), en pacientes de alto riesgo con infarto de miocardio y aquellos con enfermedad valvular o prótesis valvular cardiaca ${ }^{1,2}$. La warfarina ha sido el anticoagulante más importante en pacientes con riesgo alto y moderado para eventos cerebrovasculares (ECV), demostrando reducir la tasa de eventos isquémicos cerebrales en pacientes con la FA hasta en un $68 \%$ cuando se mantiene un International Normalized Ratio (INR) entre 2 y $3^{3}$. No obstante, su uso es limitado debido a la necesidad de monitoreo frecuente, al ajuste de dosis y el riesgo potencial de interacciones con otros medicamentos, lo cual ha dado lugar a que solo la mitad de los pacientes que se beneficiarían de ésta, la reciban ${ }^{4}$. Ante las desventajas teóricas de la warfarina, se desarrollaron nuevos medicamentos anticoagulantes con un aparente mejor perfil de seguridad ${ }^{4-8}$.
Recientemente la US Food and Drug Administration (FDA), aprobó el uso de inhibidores directos de la trombina (el dabigatran) y del factor Xa (el rivaroxaban y el apixaban), para la prevención del ECV y el embolismo sistémico en pacientes con a FA no valvular, demostrando una disminución en la mortalidad y en el número de eventos isquémicos tanto cerebrales como sistémicos, con un aumento no significativo de sangrados mayores ${ }^{9,10}$.

Adicionalmente, también han sido aprobados para el tratamiento y la profilaxis de la tromboembolia venosa y han demostrado un buen balance entre eficacia y seguridad en pacientes con cirugía ortopédica, principalmente tras cirugías de reemplazo de la cadera o de la rodilla ${ }^{1-13}$. A pesar de su reciente inclusión y disponibilidad para los pacientes afiliados al Sistema General de Seguridad Social en Salud (SGSSS), en el país aún es poca la información disponible acerca de su uso. El presente estudio buscó determinar cuál es el patrón de prescripción de los nuevos anticoagulantes orales en pacientes colombianos y su costo para el sistema de salud, pretendiendo además identificar aquellos pacientes con interacciones medicamentosas potencialmente riesgosas con el fin de ofrecer medidas para la reevaluación y ajuste de la conducta terapéutica. 


\section{Métodos}

\section{Selección y descripción de los participantes}

Se realizó un estudio observacional de corte transversal, entre el $1^{\circ}$. de febrero y el 30 de abril de 2014 sobre la utilización de nuevos anticoagulantes orales en una población aproximada de 6,5 millones de personas afiliadas al régimen contributivo del SGSSS a través de cinco Entidades Promotoras de Salud (EPS), correspondiente aproximadamente al $30,0 \%$ de la población afiliada a este régimen y al 13,5\% de la población colombiana. Se incluyeron en el estudio la totalidad de los pacientes con prescripción de nuevos anticoagulantes orales en monoterapia o en combinación con otros medicamentos durante tres meses consecutivos, residentes en alguna de las 30 ciudades más importantes del país, entre ellas: Bogotá, Barranquilla, Cali y Pereira.

El criterio de prescripción durante 3 meses consecutivos, se estableció para garantizar adherencia al tratamiento de manera más o menos estable, reflejando tolerabilidad a la medicación; mientras que se excluyeron a las personas que incumplieron las citas médicas al final del período de observación, ya que se consideró que estos individuos introducían sesgos en un estudio dirigido a describir patrones de empleo de medicamentos utilizados de manera crónica y continua.

A partir de la información sobre los consumos de medicamentos del operador logístico Audifarma, S.A, empresa que dispensa fármacos a la población objetivo, se diseñó una base de datos y se analizaron las siguientes variables:

a) Sociodemográficas: sexo, edad, ciudad de residencia.

b) Número de pacientes que reciben el dabigatran, el rivaroxaban y el apixaban, con sus dosis y se utilizó como unidad técnica de medida la dosis diaria definida y la dosis diaria definida por 1.000 habitantes y día.

c) Comedicación: 1) Antihipertensivos y diuréticos. 2) Insulinas y antidiabéticos. 3) Nitratos. 4) Analgésicos. 5) Antiarrítmicos. 6) Inotrópicos. 7) Antiulcerosos. 8) Hipolipemiantes. 9) Antidepresivos. 10) Otros antiagregantes. 11) Hormona tiroidea y antitiroideos.

Se identificaron los diagnósticos principales asociados a cada prescripción. Pero además, se utilizó la comedicación como un indicador sustituto de enfermedad crónica asociada, así: antihipertensivos y diuréticos/hipertensión arterial, antidiabéticos/diabetes mellitus, nitratos/enfermedad coronaria, analgésicos/dolor, amiodarona o propafenona/arritmias, digitálicos/insuficiencia cardiaca o fibrilación auricular, antiulcerosos/enfermedad ácido péptica, hipolipemiantes/dislipidemia, antidepresivos/depresión, hormona tiroidea/hipotiroidismo.

Por último, se revisaron las combinaciones de medicamentos que pudieran ocasionar interacciones potencialmente riesgosas con el propósito de hacer recomendaciones al ente responsable de la atención del paciente, para la corrección del posible riesgo asociado.

\section{Información técnica}

Para el análisis de los datos, se utilizó el paquete estadístico IBM SPSS Statistics, versión 22.0 (IBM, EE.UU.) para
Windows. Se emplearon las pruebas $t$ de Student o ANOVA para la comparación de variables cuantitativas y la de ji al cuadrado para las categóricas. Se aplicaron modelos de regresión logística binaria usando como variables dependientes la terapia única con nuevos anticoagulantes vs. terapia combinada y la comedicación (sí/no), y como covariables las que se asociaron significativamente con las variables dependientes en los análisis bivariados. Se determinó como nivel de significación estadística una $\mathrm{p}<0,05$.

\section{Consideraciones bioéticas}

El trabajo contó con el aval del Comité de Bioética de la Universidad Tecnológica de Pereira y se clasificó en la categoría de investigación sin riesgo según la resolución No. 8430 de 1993 del Ministerio de Salud de Colombia, respetando los principios de confidencialidad según la Declaración de Helsinki.

\section{Resultados}

Se encontró un total de 1.310 pacientes en tratamiento con nuevos anticoagulantes orales por un período mínimo de tres meses, $561(42,8 \%)$ eran mujeres y $749(57,2 \%)$ hombres, la edad media fue de $72,7 \pm 13,1$ años (rango: 18-98 años). En la fig. 1 se muestra la distribución por la edad y el sexo de este grupo de pacientes.

El agente más prescrito fue el rivaroxaban $(52,9 \%$ de los pacientes), seguido por el dabigatran y el apixaban. Los patrones de prescripción de los tres nuevos anticoagulantes orales utilizados son resumidos en la tabla 1. Mientras que los diagnósticos más comúnmente identificados se hallan en la tabla 2.

\section{Monoterapia vs. asociaciones}

Del total de pacientes del estudio, a $1.153(88,0 \%)$ se les prescribió un nuevo anticoagulante oral como monoterapia,

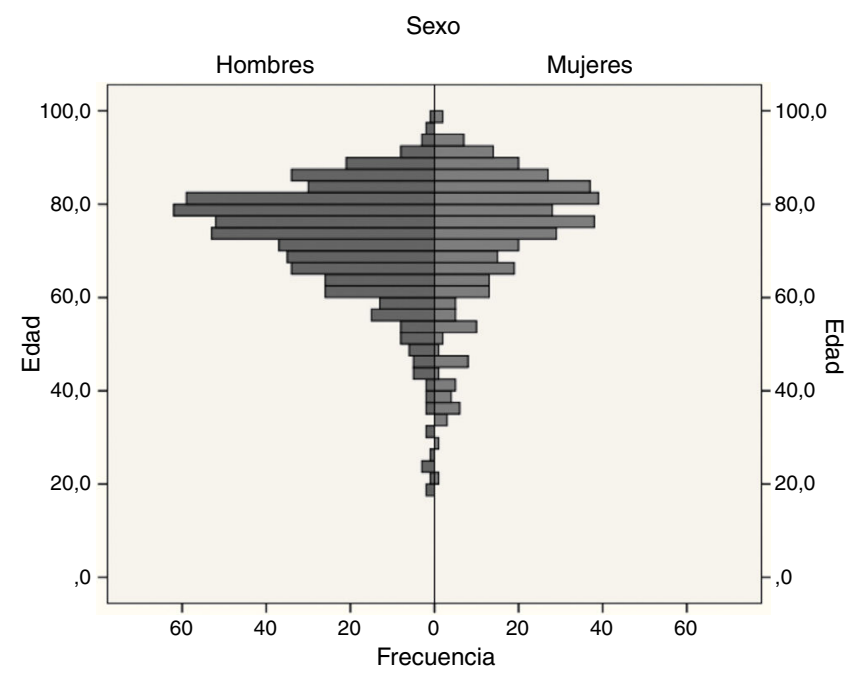

Figura 1 Distribución por edad y sexo de 1310 pacientes en tratamiento con nuevos anticoagulantes en Colombia, 2014. Fuente: autores 
Tabla 1 Patrones de prescripción de nuevos anticoagulantes en Colombia, 2014

\begin{tabular}{|c|c|c|c|c|c|c|}
\hline \multirow[t]{2}{*}{ Medicamento } & \multicolumn{2}{|c|}{ Frecuencia de prescripción } & \multicolumn{2}{|c|}{ Dosis prescrita (mg/día) } & \multirow{2}{*}{$\begin{array}{l}\text { Relación sexo } \\
(\mathrm{M}: \mathrm{F})\end{array}$} & \multirow{2}{*}{$\begin{array}{l}\text { Edad } \\
\text { Media } \pm \mathrm{DE}\end{array}$} \\
\hline & $(n=1310)$ & $(\%)$ & Media & Moda & & \\
\hline Rivaroxaban $20 \mathrm{mg}$ & 405 & 30,9 & 18,8 & 20 & $1,7: 1$ & $73,1 \pm 19,9$ \\
\hline Dabigatran $110 \mathrm{mg}$ & 390 & 29,8 & 214,1 & 220 & $1: 1$ & $80,6 \pm 15,5$ \\
\hline Rivaroxaban $15 \mathrm{mg}$ & 238 & 18,2 & 14,4 & 15 & $1,5: 1$ & $81,4 \pm 16,6$ \\
\hline Dabigatran $150 \mathrm{mg}$ & 180 & 13,7 & 295 & 300 & $1,8: 1$ & $71,8 \pm 16,4$ \\
\hline Rivaroxaban $10 \mathrm{mg}$ & 52 & 4,0 & 14,4 & 10 & $1,1: 1$ & $82,3 \pm 17,3$ \\
\hline Apixaban $5 \mathrm{mg}$ & 16 & 1,2 & 10,6 & 10 & $1: 1$ & $66,3 \pm 13,4$ \\
\hline Apixaban 2,5 mg & 15 & 1,1 & 5 & 5 & $0,4: 1$ & $81,4 \pm 6,4$ \\
\hline Dabigatran $75 \mathrm{mg}$ & 14 & 1,1 & 144,6 & 150 & $0,4: 1$ & $78,4 \pm 17,3$ \\
\hline
\end{tabular}

Tabla 2 Principales diagnósticos identificados en pacientes que reciben nuevos anticoagulantes orales en Colombia, 2014

\begin{tabular}{lll}
\hline Diagnóstico & Frecuencia & (\%) \\
\hline Fibrilación y aleteo auricular & 370 & 49,1 \\
Enfermedad cardiaca & 152 & 20,2 \\
$\quad$ isquémica/hipertensiva & & \\
Trastornos tromboembólicos & 90 & 12,0 \\
Otro trastornos del ritmo & 54 & 7,2 \\
$\quad$ Enfermedad o secuelas de & 38 & 5,0 \\
$\quad$ evento cerebrovascular & & \\
Enfermedad valvular o válvula & 12 & 1,6 \\
$\quad$ protésica & & \\
\hline
\end{tabular}

en tanto que 138 pacientes (10,5\%) recibieron la asociación de un nuevo anticoagulante más terapia antiagregante, encontrando que 12 de estos pacientes tenían formulación con antiagregacion dual y de estos, uno recibía además otro anticoagulante (la enoxaparina). Finalmente, $21(1,6 \%)$ sujetos tenían prescripción con dos anticoagulantes (un nuevo anticoagulante más una heparina). En la fig. 2 se muestra la relación monoterapia/politerapia para los tres anticoagulantes evaluados; destacando que para todos fue más probable emplearlos como monoterapia que en asociación. Las combinaciones más frecuentemente utilizadas fueron:

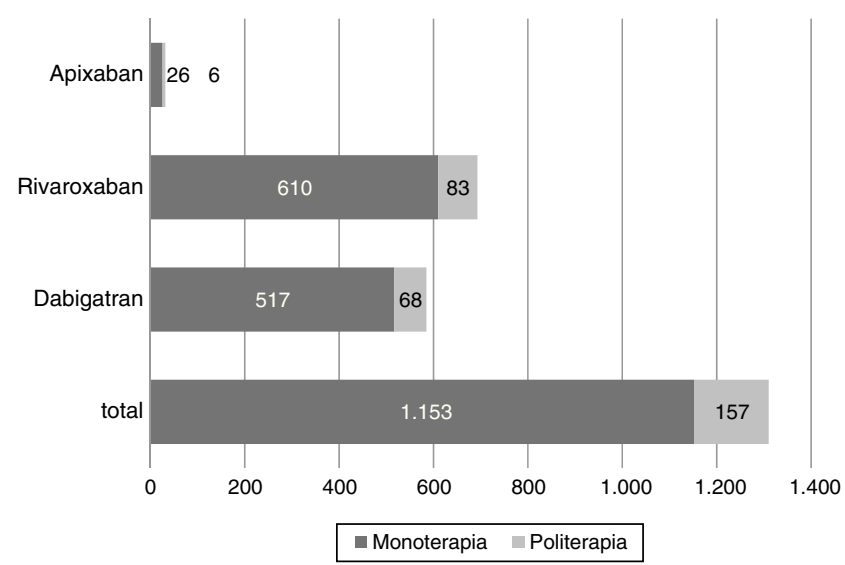

Figura 2 Frecuencia de prescripción de nuevos anticoagulantes en monoterapia o politerapia; Colombia, 2014.

Fuente: autores el rivaroxaban más ácido acetil salicílico en 68 casos $(43,3 \%$ de los pacientes), el dabigatran más ácido acetil salicílico con 51 casos $(32,5 \%)$ y el rivaroxaban más la enoxaparina en 11 sujetos $(7 \%)$.

El análisis multivariado mostró que las variables que se asociaron con un mayor riesgo de recibir terapia combinada anticoagulante fueron la comedicación con los analgésicos, las tiazidas o las estatinas (tabla 3 ). No hubo asociaciones con la edad, el sexo o la ciudad donde fuere atendido el paciente.

\section{Comedicación}

Entre los pacientes incluidos en este estudio, 1.018 (77,7\%) recibían concomitantemente uno o varios medicamentos para otras enfermedades, los cuales pueden presentar posibles interacciones con algunos de los nuevos anticoagulantes. Entre las comedicaciones más frecuentes se encontró el uso de: antiarritmicos (746 pacientes, 56,9\%), antihipertensivos (732 pacientes, 55,9\%), hipolipemiantes (627 pacientes, 47,9\%), antiulcerosos (515 pacientes, 39,3\%), analgésicos (297 pacientes, 32,7\%), antidiabéticos (197 pacientes, $15,0 \%$ ) y psicofármacos (178 pacientes, $13,6 \%)$. Se halló un total de 100 pacientes $(7,6 \%)$ distribuidos uniformemente en todas la ciudades, que recibían conjuntamente antiarrítmicos, antihipertensivos, hipolipemiantes y antidiabéticos, constituyéndose así un grupo poblacional de alto riesgo cardiovascular.

Al analizar mediante regresión logística binaria la relación con el empleo de comedicación para alguna comorbilidad se encontro que las covariables ser tratados en las ciudades de Pereira y Cartagena tuvieron una asociacion estadisticamente significativamente con un mayor riesgo de recibirla, mientras que ser menor de 65 años y ser tratado en las ciudades de Cali y Armenia disminuyen este riesgo (tabla 4).

\section{Comparación entre ciudades}

Se compararon las variables demográficas y algunos indicadores de prescripción entre las 30 ciudades colombianas incluidas en este estudio, no obstante, por razones relacionadas con el bajo número de pacientes en algunas de ellas, en la tabla 5 solo se incluyeron las ocho principales, donde reside el $79,4 \%$ de los pacientes y se hizo el mismo análisis 
Tabla 3 Análisis multivariado de las variables asociadas al tratamiento con nuevos anticoagulantes combinados con otros anticoagulantes o antiagregantes en Colombia, 2014

\begin{tabular}{|c|c|c|c|c|c|c|c|c|}
\hline & \multirow[t]{2}{*}{ B } & \multirow{2}{*}{$\begin{array}{l}\text { Error } \\
\text { estándar }\end{array}$} & \multirow[t]{2}{*}{ Wald } & \multirow[t]{2}{*}{$\mathrm{gl}$} & \multirow[t]{2}{*}{ Sig. } & \multirow[t]{2}{*}{ OR } & \multicolumn{2}{|c|}{ IC $95 \%$} \\
\hline & & & & & & & Inferior & Superior \\
\hline Inhibidores enzima convertidora angiotensina & 0,23 & 0,31 & 0,53 & 1 & 0,466 & 1,25 & 0,681 & 2,310 \\
\hline Antagonistas Receptor Angiotensina ॥ & 0,11 & 0,24 & 0,22 & 1 & 0,637 & 1,12 & 0,697 & 1,804 \\
\hline Calcioantagonistas & $-0,03$ & 0,23 & 0,01 & 1 & 0,910 & 0,97 & 0,619 & 1,532 \\
\hline B-Bloqueantes & 0,43 & 0,25 & 3,07 & 1 & 0,080 & 1,54 & 0,951 & 2,484 \\
\hline Analgésicos & 2,51 & 0,25 & 99,30 & 1 & $<0,001$ & 12,26 & 7,487 & 20,066 \\
\hline Tiazidas & 0,58 & 0,26 & 4,99 & 1 & 0,026 & 1,79 & 1,074 & 2,974 \\
\hline Antiarrítmicos & 0,48 & 0,27 & 3,16 & 1 & 0,075 & 1,61 & 0,953 & 2,718 \\
\hline Antiulcerosos anti H2 & 0,35 & 0,36 & 0,92 & 1 & 0,338 & 1,42 & 0,695 & 2,892 \\
\hline Estatinas & 0,67 & 0,23 & 8,77 & 1 & 0,003 & 1,95 & 1,254 & 3,043 \\
\hline Ezetimibe & 1,14 & 0,75 & 2,30 & 1 & 0,130 & 3,13 & 0,715 & 13,736 \\
\hline Inhibidores selectivos recaptación serotonina & 0,27 & 0,33 & 0,69 & 1 & 0,407 & 1,31 & 0,690 & 2,492 \\
\hline Recibe alguna comedicación & $-0,25$ & 0,62 & 0,16 & 1 & 0,690 & 0,78 & 0,232 & 2,635 \\
\hline Edad $<65$ años & $-0,29$ & 0,31 & 0,84 & 1 & 0,360 & 0,75 & 0,408 & 1,385 \\
\hline \multicolumn{9}{|l|}{ Ciudad donde es tratado } \\
\hline Cali & $-0,57$ & 0,32 & 3,14 & 1 & 0,077 & 0,56 & 0,300 & 1,063 \\
\hline Pereira & 0,06 & 0,26 & 0,06 & 1 & 0,809 & 1,06 & 0,641 & 1,767 \\
\hline Manizales & $-19,74$ & 4929,75 & 0,00 & 1 & 0,997 & 0,00 & 0,000 & \\
\hline Constante & $-4,00$ & 0,52 & 59,95 & 1 & 0,000 & 0,02 & & \\
\hline
\end{tabular}

Sig: nivel de significación. OR: odds ratio. IC 95\%: intervalo de confianza de $95 \%$.

Las cifras en negrita coresponden a las cifras que resultaron estadísticamente significativas.

para la sumatoria global. Se observan algunas diferencias entre las ciudades respecto al porcentaje de mujeres que reciben los nuevos anticoagulantes orales, encontrándose una mayor proporción de ellas en las ciudades de Bogotá, Cali, Palmira y menor en las ciudades restantes.

\section{Análisis económico}

El consumo de los nuevos anticoagulantes se presenta en forma de porcentaje de dosis diaria definida (DDD), para que puedan ser útiles en posteriores comparaciones. Las dosis a que están siendo empleados cada uno de los medicamentos evaluados puede verse en la tabla 1. Se encontró que, la media de consumo es de 0,16 DDD del dabigatran, 0,31 DDD del rivaroxaban y 0,01 DDD del apixaban por 1.000 habitantes/día.

El coste total de la dispensación de los nuevos anticoagulantes a la población afiliada objeto de estudio por mes fue de COP $\$ 148.263 .960$ pesos (US $\$ 74.243 ; € 53.914$ ) y por año COP $\$ 1.779 .167 .520$ (US $\$ 890.920$; $€ 646.970$ ). El costo del dabigatran por año se estimó en $\$ 458.686 .800$ (US \$229.688; $€ 166.795)$, mientras del rivaroxaban en COP $\$ 1.275 .840 .720$ (US $\$ 638.879 ; € 463.942$ ) y del apixaban en COP $\$ 44.640 .000$ (US $\$ 22.354$; $€ 16.233$ ). El costo por 1.000 habitantes y día para el dabigatran COP $\$ 334,6$ (US $\$$ 0,$16 ; 0,12 €$ ); para el rivaroxaban COP $\$ 930,7$ (US $\$ 0,46$; $0,34 €$ ) y para el apixaban COP $\$ 32,6$ (US $\$ 0,016 ; 0,01 €$ ).

Tabla 4 Análisis multivariado de las variables asociadas al tratamiento con nuevos anticoagulantes y comedicación en Colombia, 2014

\begin{tabular}{|c|c|c|c|c|c|c|c|c|}
\hline & \multirow[t]{2}{*}{ B } & \multirow{2}{*}{$\begin{array}{l}\text { Error } \\
\text { estándar }\end{array}$} & \multirow[t]{2}{*}{ Wald } & \multirow[t]{2}{*}{$\mathrm{gl}$} & \multirow[t]{2}{*}{ Sig. } & \multirow[t]{2}{*}{ OR } & \multicolumn{2}{|c|}{ IC $95 \%$} \\
\hline & & & & & & & Inferior & Superior \\
\hline Edad $<65$ años & $-0,56$ & 0,18 & 9,44 & 1 & 0,002 & 0,569 & 0,397 & 0,815 \\
\hline \multicolumn{9}{|c|}{ Ciudad donde es tratado } \\
\hline Bogotá & 0,29 & 0,20 & 2,17 & 1 & 0,141 & 1,333 & 0,909 & 1,953 \\
\hline Cali & $-1,15$ & 0,17 & 43,24 & 1 & $<0,001$ & 0,317 & 0,225 & 0,447 \\
\hline Pereira & 2,05 & 0,47 & 18,88 & 1 & $<0,001$ & 7,746 & 3,077 & 19,501 \\
\hline Barranquilla & 0,70 & 0,40 & 3,12 & 1 & 0,077 & 2,020 & 0,926 & 4,403 \\
\hline Cartagena & 1,86 & 0,73 & 6,46 & 1 & 0,011 & 6,449 & 1,532 & 27,155 \\
\hline Armenia & $-0,96$ & 0,31 & 9,41 & 1 & 0,002 & 0,382 & 0,207 & 0,707 \\
\hline Constante & 1,43 & 0,13 & 126,27 & 1 & 0,000 & 4,196 & & \\
\hline
\end{tabular}

Sig: nivel de significación. OR: odds ratio. IC 95\%: intervalo de confianza de $95 \%$.

Las cifras en negrita coresponden a las cifras que resultaron estadísticamente significativas. 
Tabla 5 Comparación de algunas variables demográficas e indicadores de prescripción de nuevos anticoagulantes y relación entre la dosis media administrada y la dosis diaria definida entre ocho ciudades colombianas, 2014

\begin{tabular}{|c|c|c|c|c|c|c|c|c|c|}
\hline Variables & $\begin{array}{l}\text { Bogotá } \\
n=313\end{array}$ & $\begin{array}{l}\text { Cali } \\
n=266\end{array}$ & $\begin{array}{l}\text { Pereira } \\
n=157\end{array}$ & $\begin{array}{l}\text { Palmira } \\
\mathrm{n}=84\end{array}$ & $\begin{array}{l}\text { Barranquilla } \\
\mathrm{n}=67\end{array}$ & $\begin{array}{l}\text { Manizales } \\
\mathrm{n}=54\end{array}$ & $\begin{array}{l}\text { Armenia } \\
n=51\end{array}$ & $\begin{array}{l}\text { Cartagena } \\
\mathrm{n}=49\end{array}$ & $\begin{array}{l}\text { Global } \\
n=1310\end{array}$ \\
\hline Edad promedio & 73,1 & 75,6 & 74,5 & 76,3 & 71,0 & 67,3 & 74,9 & 68,7 & 72,7 \\
\hline Mujeres (\%) & 55,9 & 52,2 & 38,2 & 54,7 & 25,3 & 24,0 & 23,5 & 20,4 & 42,8 \\
\hline Monoterapia (\%) & 88,1 & 94,3 & 80,9 & 88,0 & 83,5 & 100,0 & 86,2 & 85,7 & 88,0 \\
\hline \multicolumn{10}{|l|}{ Comedicación (\%) } \\
\hline Cardiovascular & 74,1 & 50,0 & 84,7 & 70,2 & 85,0 & 75,9 & 58,8 & 89,7 & 69,9 \\
\hline Diabetes Mellitus & 14,3 & 10,9 & 22,2 & 19,0 & 11,9 & 11,1 & 15,6 & 18,3 & 15,0 \\
\hline Dislipidemia & 54,3 & 28,9 & 66,2 & 46,4 & 44,7 & 59,2 & 39,2 & 65,3 & 47,9 \\
\hline \multicolumn{10}{|c|}{ Tasa de prescripción (\%) } \\
\hline Apixaban 2,5 mg & 6,0 & 46,6 & 6,0 & 26,6 & 0 & 0 & 6,0 & 0 & 1,1 \\
\hline Apixaban $5 \mathrm{mg}$ & 37,5 & 37,5 & 0 & 0 & 12,5 & 0 & 0 & 0 & 1,2 \\
\hline Dabigatran $75 \mathrm{mg}$ & 57,1 & 7,1 & 0 & 7,1 & 0 & 0 & 0 & 7,1 & 1,1 \\
\hline Dabigatran $110 \mathrm{mg}$ & 35,6 & 17,7 & 8,4 & 8,2 & 2,3 & 2,1 & 2,1 & 4,1 & 29,8 \\
\hline Dabigatran $150 \mathrm{mg}$ & 21,7 & 31,1 & 7,2 & 2,8 & 3,9 & 2,8 & 4,4 & 5,0 & 13,7 \\
\hline Rivaroxaban $10 \mathrm{mg}$ & 25,0 & 7,7 & 34,6 & 3,8 & 5,8 & & 7,7 & 1,9 & 4,0 \\
\hline Rivaroxaban $15 \mathrm{mg}$ & 17,6 & 16,4 & 18,1 & 2,9 & 6,3 & 9,7 & 5,9 & 2,5 & 18,2 \\
\hline Rivaroxaban $20 \mathrm{mg}$ & 16,0 & 20,7 & 12,1 & 8,1 & 7,6 & 4,4 & 3,4 & 4,0 & 30,9 \\
\hline \multicolumn{10}{|c|}{ Relación entre la dosis media y la DDD } \\
\hline Apixaban 2,5 mg & 1,00 & 1,00 & 1,00 & 1,00 & 1,00 & 1,00 & 1,00 & 1,00 & 1,00 \\
\hline Apixaban $5 \mathrm{mg}$ & 2,00 & 2,30 & 0 & 0 & 2,00 & 0 & 0 & 0 & 2,10 \\
\hline Dabigatran $75 \mathrm{mg}$ & 0,63 & 0,68 & 0 & 0,68 & 0 & 0 & 0 & 0,68 & 0,67 \\
\hline Dabigatran $110 \mathrm{mg}$ & 0,98 & 1,00 & 0,98 & 0,95 & 1,00 & 0,94 & 0,93 & 1,00 & 0,97 \\
\hline Dabigatran $150 \mathrm{mg}$ & 1,34 & 1,36 & 1,36 & 1,22 & 1,36 & 1,10 & 1,36 & 1,28 & 1,30 \\
\hline Rivaroxaban $10 \mathrm{mg}$ & 1,58 & 1,50 & 1,38 & 2,00 & 1,00 & & 1,25 & 2,00 & 1,53 \\
\hline Rivaroxaban $15 \mathrm{mg}$ & 1,50 & 1,40 & 1,43 & 1,40 & 1,40 & 1,46 & 1,40 & 1,50 & 1,44 \\
\hline Rivaroxaban $20 \mathrm{mg}$ & 1,86 & 1,86 & 1,86 & 1,92 & 1,86 & 1,86 & 2,00 & 1,86 & 1,88 \\
\hline
\end{tabular}

DDD: Dabigatran: $220 \mathrm{mg}$. Rivaroxaban: $10 \mathrm{mg}$. Apixaban: $5 \mathrm{mg}$

\section{Discusión}

El presente estudio determinó el patrón de prescripción y los costos de los nuevos anticoagulantes orales en pacientes afiliados al SGSSS de Colombia, encontrando que estos son más frecuentemente formulados en monoterapia y que el de mayor prescripción es el rivaroxaban, siendo a su vez el más costoso de dichos medicamentos. Este estudio brinda información válida a los administradores del sistema de salud para establecer medidas efectivas en la atención de los pacientes que requieren anticoagulación.

La relación por sexo mostró un ligero predominio masculino, similar a lo registrado en grandes estudios que han evaluado el uso de nuevos anticoagulantes orales en pacientes con $\mathrm{FA}^{14-17}$ y que puede guardar relación con un riesgo ligeramente mayor de desarrollar dicha condición en hombres que en mujeres ${ }^{18,19}$, pero que no ha sido establecido para el TEV. En este estudio la edad media fue de 72,7 años, dato que puede asociarse a que tanto la fibrilación auricular, principal indicación de la terapia anticoagulante, como el TEV son condiciones que aumentan su frecuencia con la edad $^{19-21}$.

Diferentes estudios realizados en pacientes con TEV y FA han comparado el rivaroxaban, el dabigatran o el apixaban con la warfarina, mostrando que los nuevos anticoagulantes orales no son inferiores a la warfarina en términos de prevención de ECV, embolismo sistémico o en el tratamiento del TEV, y en algunos casos pueden tener menores tasas de sangrado intracraneal ${ }^{15-17,22}$, aunque ha surgido una preocupación debido a reportes recientes de aumento en el riesgo de hemorragia gastrointestinal ${ }^{23-25}$. Datos de dos diferentes estudios sugieren que el apixaban puede ser el más seguro de los nuevos anticoagulantes orales en el tratamiento de TEV, sin embargo, son necesarios más estudios que evalúen la seguridad de estos medicamentos ${ }^{26,27}$. En nuestro estudio, el agente más prescrito fue el rivaroxaban $(52,9 \%)$, seguido del dabigatran $(44,7 \%)$ y el apixaban $(2,4 \%)$.

Al realizar el análisis de costos se evidencia que el consumo del rivaroxaban por paciente por año tiene un costo de COP \$1.841.040 para el SGSSS, mientras los datos arrojados por el National Health System (NHS) en el año 2012 mostraron un costo estimado por paciente por año de COP \$ 267.904 del tratamiento con la warfarina, incluidos los exámenes de monitoreo ${ }^{28}$.

Del total de pacientes del estudio, el $12,1 \%$ presentaron asociación de un nuevo anticoagulante y algún antiagregante u otro anticoagulante. En el caso de la terapia combinada con dos anticoagulantes, encontrada en 21 de estos pacientes, es posible que se deba a la transición entre un tipo de medicamento y otro durante el período de observación y no a una doble terapia contemporánea. Por otro lado, la terapia combinada con antiagregantes en 138 pacientes, es 
aconsejada cuando el riesgo tromboembólico es elevado y el de sangrado es bajo ${ }^{25,29}$. Una revisión sistemática reciente demostró que aunque la combinación de ácido acetil salicílico más clopidogrel es más efectivo que la primera en monoterapia para prevención de ictus en pacientes con ECV o ataque isquémico transitorio previo, también acarrean un mayor riesgo de sangrado (RR: 1,75; IC: 95\%: 1,48-2,05) 30. En el mismo sentido, un metaanálisis y varios estudios han revelado que en comparación con la monoterapia con el clopidogrel, la terapia dual tiene un mayor riesgo de hemorragia intracraneal (RR: 1,46; IC: 95\%: 1,17-1,82) ) $^{31-33}$.

De acuerdo a las guías de manejo de la fibrilación auricular y algunos estudios conducidos para tratamiento del TEV, las dosis medias de los tres nuevos anticoagulantes mostradas en este estudio, se encuentran en el rango de dosis recomendadas, considerando que para el apixaban y el rivaroxaban las dosis empleadas en algunos de los estudios para tratamiento del TEV son superiores respecto a la recomendada para la $\mathrm{FA}^{19,26}$.

La evaluación a partir de la DDD, mostró que el dabigatran $110 \mathrm{mg}$ y el apixaban $2,5 \mathrm{mg}$, fueron los únicos agentes para los que la dosis empleada es la misma establecida por la OMS para su indicación principal, mientras que las dosis suministradas de los otros nuevos anticoagulantes $y$ otras presentaciones fueron superiores a las recomendadas, con una proporción de DDD calculada en un rango de 1,3-2,1. Por su parte, la DDD del dabigatran $75 \mathrm{mg}$, fue inferior a lo establecido $(0,67)$. No se encontraron estudios que establecieran relaciones porcentuales de las DDD de los nuevos anticoagulantes orales por mil habitantes y día.

La hipertensión arterial es la comorbilidad más frecuente en esta cohorte de pacientes $(55,9 \%)$ y es ampliamente reconocida como factor de riesgo para el desarrollo de trastornos del ritmo ${ }^{18,19,33-35}$ y de eventos tromboembólicos en el paciente con la $\mathrm{FA}^{19,22,33,36-38}$.

La dislipidemia y la diabetes mellitus fueron condiciones frecuentes en este grupo de pacientes $(47,9 \%$ y $15,0 \%$, respectivamente) $)^{35,36}$. Dado que un importante número de pacientes padece simultáneamente las cuatro patologías mencionadas (FA, hipertensión arterial, diabetes mellitus, dislipidemia), suponen un grupo de muy alto riesgo cardiovascular, sobre el cual deben realizarse intervenciones de prevención que disminuyan la probabilidad de eventos coronarios, cerebrovasculares, falla cardiaca y otras complicaciones $^{39}$. Por otra parte, es importante tener precaución con las posibles interacciones medicamentosas debido a que se ha demostrado un aumento en el riesgo de sangrado en pacientes que consumen el rivaroxaban o el dabigatran junto con inhibidores de la glicoproteína P como el verapamilo y la amiodarona ${ }^{40}$.

Las diferencias encontradas en cuanto a la frecuencia de prescripción de los diferentes anticoagulantes orales entre las diferentes ciudades colombianas incluidas en el estudio, evidencian un resultado frecuente en los estudios de farmacoepidemiología, explicado por la variabilidad en la atención médica, en especial en los hábitos de prescripción subyacentes a las diferentes maneras de ejercer la práctica clínica $^{41}$. Estas variaciones, de importancia por sus implicaciones médicas, sociales y económicas, se relacionan con características de los pacientes (edad, sexo, comorbilidad) y con estilos de práctica profesional que también resultan de factores personales y de la formación y capacitación que tuvieron los médicos ${ }^{41}$.

Algunas limitaciones en la interpretación de ciertos resultados son reconocidas, sin embargo, estas pueden solucionarse con el desarrollo de estudios afines a la utilización de nuevos anticoagulantes orales donde se evalué, entre otros, los intervalos de dosis empleados, la incidencia de reacciones adversas asociadas al medicamento, la adherencia y efectividad del tratamiento y la pertinencia de la medicación. Debido a que se trata de una población con características comunes de aseguramiento y atención, las conclusiones solo pueden ser extrapolables a poblaciones con condiciones similares.

\section{Conclusiones}

A partir de los patrones de prescripción encontrados puede afirmarse que, la formulación de nuevos anticoagulantes orales se realiza principalmente en monoterapia. Dado que un importante porcentaje de pacientes presenta comedicación de un anticoagulante con un antiagregante, debe balancearse el beneficio brindado con esta terapia contra el riesgo de hemorragia, especialmente en adultos mayores.

El análisis de costos mostró que de los tres nuevos anticoagulantes orales evaluados en este estudio, el rivaroxaban corresponde al fármaco que más costos por año genera, de la misma manera representa el mayor costo por 1.000 habitantes y día, mientras que el uso de otros medicamentos, como la warfarina, generan menor carga económica.

Por otro lado, dado que la combinación de anticoagulantes y antiagregantes implican un mayor riesgo de sangrado, debe balancearse el riesgo-beneficio en cada caso particular y acoger las pautas de dosificación validadas en ensayos clínicos que permitan su adecuada utilización y disminución de costos para el SGSSS. Es necesario establecer acciones educativas dirigidas al personal médico buscando su actualización y aprendizaje permanente en terapéutica, así como promover el adecuado uso de medicamentos y sus asociaciones.

\section{Responsabilidades éticas}

Protección de personas y animales. Los autores declaran que para esta investigación no se han realizado experimentos en seres humanos ni en animales.

Confidencialidad de los datos. Los autores declaran que en este artículo no aparecen datos de pacientes.

Derecho a la privacidad y consentimiento informado. Los autores declaran que en este artículo no aparecen datos de pacientes.

\section{Fuentes de financiación}

El trabajo recibió financiación de la Universidad Tecnológica de Pereira y de Audifarma S.A. 


\section{Conflicto de intereses}

Los autores expresan que no existe ningún conflicto de intereses.

\section{Agradecimientos}

Los autores agradecen a Viviana Andrea Orozco, Soffy Claritza López y Andrea Orozco por su participación en la elaboración de la base de datos.

\section{Bibliografía}

1. Ageno W, Gallus AS, Wittkowsky A, Crowther M, Hylek EM, Palareti G, American College of Chest Physicians. Oral anticoagulant therapy: Antithrombotic Therapy and Prevention of Thrombosis, 9 th ed: American College of Chest Physicians Evidence-Based Clinical Practice Guidelines. Chest. 2012;141 2 Suppl:e44S-88S.

2. Wigle P, Hein B, Bloomfield HE, Tubb M, Doherty M. Updated guidelines on outpatient anticoagulation. Am Fam Physician. 2013;87(8):556-66.

3. Gutierrez C, Blanchard DG. Atrial fibrillation: diagnosis and treatment. Am Fam Physician. 2011;83:61-8.

4. Xu B, Whitbourn R. Novel anticoagulants for non-valvular atrial fibrillation. Heart Lung Circ. 2012;21(8):463-7.

5. Potpara TS, Lip GY. Novel oral anticoagulants in nonvalvular atrial fibrillation. Best Pract Res Clin Haematol. 2013;26(2):115-29.

6. Kansal AR, Zheng Y, Pokora T, Sorensen SV. Cost-effectiveness of new oral anticoagulants in the prevention of stroke in patients with atrial fibrillation. Best Pract Res Clin Haematol. 2013;26(2):225-37.

7. Riva N, Donadini MP, Bozzato S, Ageno W. Novel oral anticoagulants for the prevention of venous thromboembolism in surgical patients. Thromb Res. 2013;131 Suppl 1:S67-70.

8. Little JW. New oral anticoagulants: will they replace warfarin? Oral Surg Oral Med Oral Pathol Oral Radiol. 2012;113(5):575-80.

9. Capodanno D, Capranzano P, Giacchi G, Calvi V, Tamburino C. Novel oral anticoagulants versus warfarin in non-valvular atrial fibrillation: a meta-analysis of 50,578 patients. Int J Cardiol. 2013;167(4):1237-41.

10. O’Dell KM, Igawa D, Hsin J. New oral anticoagulants for atrial fibrillation: a review of clinical trials. Clin Ther. 2012;34:894-901.

11. McManus R, Fitzmaurice D, Murray E, Taylor C. Thromboembolism. Am Fam Physician. 2012;85(2):188-90.

12. Gómez-Outes A, Terleira-Fernández Al, Suárez-Gea ML, VargasCastrillón E. Dabigatran, rivaroxaban, or apixaban versus enoxaparin for thromboprophylaxis after total hip or knee replacement: systematic review, meta-analysis, and indirect treatment comparisons. BMJ. 2012;344:e3675.

13. Falck-Ytter Y, Francis CW, Johanson NA, Curley C, Dahl OE, Schulman S, et al., American College of Chest Physicians. Prevention of VTE in orthopedic surgery patients: Antithrombotic Therapy and Prevention of Thrombosis, 9 th ed: American College of Chest Physicians Evidence-Based Clinical Practice Guidelines. Chest. 2012;141(2):e278S-325S.

14. Patel MR, Mahaffey KW, Garg J, Pan G, Singer DE, Hacke W, et al., ROCKET AF Investigators. Rivaroxaban versus warfarin in nonvalvular atrial fibrillation. $N$ Engl J Med. 2011;365(10):883-91.

15. Connolly SJ, Ezekowitz MD, Yusuf S, Eikelboom J, Oldgren J, Parekh A, et al., RE-LY Steering Committee and Investigators. Dabigatran versus warfarin in patients with atrial fibrillation. $\mathrm{N}$ Engl J Med. 2009;361(12):1139-51.
16. Granger CB, Alexander JH, McMurray JJ, Lopes RD, Hylek EM, Hanna M, et al., ARISTOTLE Committees and Investigators. Apixaban versus warfarin in patients with atrial fibrillation. $\mathrm{N}$ Engl J Med. 2011;365(11):981-92.

17. Connolly SJ, Eikelboom J, Joyner C, Diener HC, Hart R, Golitsyn $\mathrm{S}$, et al., AVERROES Steering Committee and Investigators. Apixaban in patients with atrial fibrillation. $N$ Engl J Med. 2011;364(9):806-17.

18. Marcus GM, Alonso A, Peralta CA, Lettre G, Vittinghoff E, Lubitz SA, et al., Candidate-Gene Association Resource (CARe) Study. European ancestry as a risk factor for atrial fibrillation in African Americans. Circulation. 2010;122(20):2009-15.

19. January CT, Wann LS, Alpert JS, Calkins H, Cigarroa JE, Cleveland JC Jr, et al., ACC/AHA Task Force Members. 2014 AHA/ACC/HRS guideline for the management of patients with atrial fibrillation: executive summary: a report of the American College of Cardiology/American Heart Association Task Force on practice guidelines and the Heart Rhythm Society. Circulation. 2014;130(23):2071-104.

20. Rao MP, Pokorney SD, Granger CB. Atrial fibrillation: a review of recent studies with a focus on those from the duke clinical research institute. Scientifica (Cairo). 2014;2014: 901586.

21. Konstantinides SV, Torbicki A, Agnelli G, Danchin N, Fitzmaurice $D$, Galiè N, et al., Task Force for the Diagnosis and Management of Acute Pulmonary Embolism of the European Society of Cardiology (ESC). 2014 ESC guidelines on the diagnosis and management of acute pulmonary embolism. Eur Heart J. 2014;35(43):3033-69, 3069-3069k.

22. Albert NM. Use of novel oral anticoagulants for patients with atrial fibrillation: systematic review and clinical implications. Heart Lung. 2014;43(1):48-59.

23. Gómez-Outes A, Terleira-Fernández Al, Calvo-Rojas G, SuárezGea ML, Vargas-Castrillón E. Dabigatran, Rivaroxaban, or Apixaban versus Warfarin in Patients with Nonvalvular Atrial Fibrillation: A Systematic Review and Meta-Analysis of Subgroups. Thrombosis. 2013;2013:640723.

24. Levy JH, Levi M. New oral anticoagulant-induced bleeding: clinical presentation and management. Clin Lab Med. 2014;34(3):575-86.

25. Holster IL, Valkhoff VE, Kuipers EJ, Tjwa ET. New oral anticoagulants increase risk for gastrointestinal bleeding: a systematic review and meta-analysis. Gastroenterology. 2013;145(1), 105112.e15.

26. Agnelli G, Becattini C, Franco L. New oral anticoagulants for the treatment of venous thromboembolism. Best Pract Res Clin Haematol. 2013;(2):151-61.

27. Kang N, Sobieraj DM. Indirect treatment comparison of new oral anticoagulants for the treatment of acute venous thromboembolism. Thromb Res. 2014;133(6):1145-51.

28. Kerr M. Cost and benefits of Antithrombotic therapy in atrial fibrilation in England: an economic analysis based on GRASP-AF. National Health System (NHS). 2014. fecha de consulta: abril 24 del 2015. Disponible en: http://www.nhsiq.nhs. uk/media/2566750/af_economic_analysis_final.pdf

29. Stefanini GG, Holmes DR Jr. Drug-eluting coronary-artery stents. N Engl J Med. 2013;368(3):254-65.

30. Huang Y, Li M, Li JY, Li M, Xia YP, Mao L, et al. The efficacy and adverse reaction of bleeding of clopidogrel plus aspirin as compared to aspirin alone after stroke or TIA: a systematic review. PLoS One. 2013;8(6):e65754.

31. Hong KS. Dual antiplatelet therapy after noncardioembolic ischemic stroke or transient ischemic attack: pros and cons. J Clin Neurol. 2014;10(3):189-96.

32. Dewilde WJ, Janssen PW, Verheugt FW, Storey RF, Adriaenssens T, Hansen ML, et al. Triple therapy for atrial fibrillation and percutaneous coronary intervention: a contemporary review. J Am Coll Cardiol. 2014;64(12):1270-80. 
33. Dzeshka MS, Antithrombotic Lip GY. Anticoagulant Therapy for Atrial Fibrillation. Cardiol Clin. 2014;32(4):585-99.

34. Guérin A, Lin J, Jhaveri M, Wu EQ, Yu AP, Cloutier $M$, et al. Outcomes in atrial fibrillation patients on combined warfarin \& antiarrhythmic therapy. Int J Cardiol. 2013;167(2):564-9.

35. Gorczyca-Michta I, Wożakowska-Kapłon B, Tomasik E. Prevalence and predisposing conditions for atrial fibrillation in hospitalised patients with hypertension. Kardiol Pol. 2013;71(4):352-8.

36. Vyssoulis G, Karpanou E, Adamopoulos D, Kyvelou SM, Tzamou V, Michaelidis A, et al. Metabolic syndrome and atrial fibrillation in patients with essential hypertension. Nutr Metab Cardiovasc Dis. 2013;23(2):109-14.

37. Jiří Widimský. Arterial hypertension and atrial fibrillation: Selecting antihypertensive therapy. Cor et Vasa. 2012;54(4):e248-52.
38. Lane DA, Lip GY. Use of the CHA2DS2-VASC and HASBLED scores to aid decision making for thromboprophylaxis in nonvalvular atrial fibrillation. Circulation. 2012;126(7): $860-5$.

39. Koju R, Humagain S, Khanal K. Association of cardiovascular risk factors and coronary artery lesion among coronary artery disease patients. Kathmandu Univ Med J (KUMJ). 2014;12(46):137-40.

40. Aristizábal J, Restrepo A, Uribe W, Medina E, Marín JE, Velásquez JE, et al. Consideraciones prácticas para el uso de los nuevos anticoagulantes orales. Rev Colomb Cardiol. 2012;19(3):135-41.

41. Machado-Alba JE, Moncada JC, Mesa G. Prescription patterns for antilipidemic drugs in a group of Colombian patients. Rev Panam Salud Publica. 2008;23(3):179-87. 